VeritasE Scientia

Vol. 8. N 1.

Enero - Junio del 2019.

ISSN Edición Online: 2617-0639

https://doi.org/10.47796/ves.v8i1.112

\title{
GESTION DE RIESGOS Y SU INFLUENCIA EN LAS ACCIONES REALIZADAS EN LOS PUESTOS DE CONTROL EN LA INTENDENCIA DE ADUANA DE TACNA, PERIODO 2016
}

\author{
RISK MANAGEMENT AND ITS INFLUENCE ON THE ACTIONS CARRIED OUT AT THE CONTROL POSTS \\ AT THE CUSTOMS ATTENDANCE OF TACNA, PERIOD 2016
}

Néstor Edgardo Meza Falla ${ }^{1}$
Juan Aranibar Ocola ${ }^{2}$

Presentado: $18 / 04 / 2019$

Aceptado: $17 / 06 / 2019$

Publicado online:17/07/2019

\section{RESUMEN}

El desarrollo del presente trabajo de investigación se enfocó a los aspectos relacionados a una adecuada Gestión de riesgos, como herramienta para minimizar la posibilidad de error que se pueda presentar en las acciones de control, teniendo en cuenta que no se tiene intención de realizar un acto ilícito hasta que se demuestre lo contrario. El presente trabajo de investigación es no experimental porque se obtienen datos directamente de la realidad objeto de estudio, asimismo es descriptivo, luego explicativa y finalmente correlacionada de acuerdo a la finalidad de la investigación. El área de investigación está enmarcada en el control aduanero, estableciendo cómo la gestión de riesgos influye en las acciones realizadas en los puestos de control de la intendencia de Aduana de Tacna, a través del nivel estratégico, nivel táctico y nivel operativo. Así mismo se viene observando la falta de acciones de control por parte de la Intendencia de Aduana, ya que es necesaria la inspección de mercancías a fin de poder controlar el tránsito de bienes de acuerdo a las normativas vigentes, la inmovilización e incautación no tiene un control adecuado por la falta de medidas de seguridad y también las acciones relacionadas al Control Operativo muchas veces no cuentan con el apoyo logístico necesario para la realización de dichas actividades de manera óptima. Por lo que finalmente se llega a la conclusión de que la implementación de una adecuada Gestión de Riesgos influye significativamente en las acciones realizadas en los Puestos de Control de la intendencia de Aduana de Tacna, periodo 2016.

Palabras Claves: Gestión de riesgos, acciones de control, inspección.

\footnotetext{
${ }^{1}$ Magíster en gestión y políticas públicas

2 Doctor, Jefe de la Oficina de Contabilidad de la Universidad Privada de Tacna
} 


\section{ABSTRACT}

The development of this research work is focused on the aspects related to proper risk management, as a tool to minimize the possibility of error that may occur in control actions, taking into account that there is no intention to perform an act illicit until proven otherwise. The present research work is non-experimental because data are obtained directly from the reality under study, it is also descriptive, then explanatory and finally correlated according to the purpose of the research. The research area is framed in the customs control, establishing how the risk management influences the actions of control posts in the Tacna customs intendancy, through the strategic level, tactical level and operational level.

Likewise, the lack of control actions by the customs administration has been observed, since the inspection of merchandise is necessary in order to be able to control the transit of goods according to the regulations in force, the immobilization and seizure does not have a adequate control for the lack of security measures and also operative control often do not have the necessary logistical support. So finally it is concluded that the implementation of adequate Risk Management significantly influences the actions taken in the Control Posts of the Customs office of Tacna, 2016 period.

Key words: Risk management, control actions, inspection.

\section{INTRODUCCIÓN}

En el contexto nacional, se viene observando que la Superintendencia Nacional de Aduanas y Administración Tributaria (SUNAT) no cuenta con una adecuada gestión de riesgos, lo que genera algunas deficiencias relacionadas con la inspección de mercancías, inmovilización e incautación y el control operativo, evidenciando la falta de acción de los puestos de control, existiendo la necesidad de que los esfuerzos de las aduanas se orienten al cumplimiento de la normatividad. La gestión de riesgo como método sistemático permite identificar, evaluar y controlar los eventuales sucesos negativos y sus consecuencias. En la gestión de riesgos se puede distinguir tres aspectos: la probabilidad de ocurrencia, la magnitud del impacto y el daño que puede causar al estado. Un adecuado control permitirá optimizar la recaudación con el incremento de ingresos tributarios y por ende habrá un buen desarrollo local, y se podrá cubrir las expectativas de la población; la investigación planteada contribuirá a evaluar las Acciones de control en los puestos de la intendencia, a través de la gestión de riesgos aduaneros, mejorando los procedimientos de recaudación y control.

Teniendo en cuenta que la Superintendencia Nacional de Aduanas y Administración Tributaria controla las diferentes operaciones sobre el traslado de bienes, actualmente tiene que lidiar con un problema social que se encuentra en crecimiento con el pasar del tiempo que es el "Contrabando" y los delitos aduaneros en general. Para poder contrarrestar esta problemática, el control aduanero se debe aplicar basado en la Gestión de Riesgos, con el fin de minimizar la posibilidad de error que se pueda presentar en las acciones de control, teniendo en cuenta que no se tiene intención de realizar un acto ilícito hasta que se demuestre lo contrario. Existe la necesidad de que los esfuerzos de las aduanas se orienten a evitar que se incumpla con la normatividad vigente y se perjudique a los intereses fiscales, económicos, la salud y la seguridad de los ciudadanos y que los usuarios no tengan inconveniente para trasladar y/o retirar sus 
mercancías de los recintos aduaneros. Al usar la gestión del riesgo como método sistemático para identificar, evaluar y controlar los eventuales sucesos negativos y sus consecuencias, la aduana puede cumplir sus deberes legales. En la Gestión de Riesgos se puede distinguir tres aspectos: La probabilidad de ocurrencia, la magnitud del impacto y el daño que puede causar al Estado. El proceso de la Gestión de Riesgos, se inicia con la identificación de las áreas de riesgo de acuerdo al contexto aduanero. Una vez identificados los riesgos, estos son evaluados y se le asigna un tratamiento a cada caso particular de riesgo. Finalmente se termina en la evaluación y monitoreo para empezar de nuevo el ciclo. Un componente esencial de la Gestión de Riesgos, sobre todo en la parte del análisis y evaluación del riesgo, es el uso de los perfiles de riesgo, a través de los cuales se evalúa el nivel de riesgo de las declaraciones que ingresan por la aduana y se define el tratamiento que se le va a dar en cada caso particular. Existen tres momentos para uso de perfiles de riesgo en el control aduanero: Previo, Concurrente y Posterior. En el Área Base seleccionada para la investigación son: Control Fronterizo "Santa Rosa", Centro de Control Aduanero "Tomasiri" y Puesto de Control Aduanero "Vila Vila" la inspección que se realiza de manera aleatoria por parte del inspector no despierta muchas sospechas por lo que no se revisa detenidamente la mercancía. Dentro de los despachos aduaneros, al momento de aplicarse medidas preventivas como son la incautación o inmovilización de mercancías, los despachadores de aduana o usuarios, suelen argumentar como defensa frente a la aplicación de dichas medidas, el desconocimiento o cambio repentino de la normatividad en la materia. El personal de la División de Control Operativo, ejercen un control aduanero sin contar con equipos tecnológicos y herramientas adecuados para un óptimo control y prevención de infracciones y de delitos aduaneros.

Chacón C. y Chávez M. (2010), concluye que siendo que el hacinamiento de mercancías en el Departamento de Almacén de Mercancías de la Intendencia de Aduana de Tacna es el principal problema materia del presente trabajo de investigación, se ha demostrado que existen diferentes medios y mecanismos coordinados que pueden generar el incremento en la rotación del stock de bienes en los inventarios de almacenes. La información es la materia prima más importante para agilizar la eliminación de los bienes del almacén. Por lo tanto, el Jefe del Departamento de Almacén debe concentrar los esfuerzos de su equipo en la gestión permanente de la información, así como en las Organizaciones Orgánicas de la Intendencia Aduanera y en las organizaciones estatales que se encargan de la evaluación de los documentos relacionados con el delito y el crimen de contrabando. Otro aspecto a resaltar está relacionado con la selección de personal según el perfil deseado por el área de usuario. De acuerdo con la recomendación, la asignación de recursos humanos, no se puede hacer una tarea que se adapte a las necesidades de los usuarios que no están familiarizados con el medio ambiente. De acuerdo a Chacón C y Chávez M. indican que, en la administración de almacenes, se debe realizar cualquier tipo de designación arbitraria de recursos. La planificación y ejecución de acciones de mejora para la disposición de los bienes da como resultado una gestión eficiente de la gestión del almacén. Estos son aspectos que permiten un aumento en la rotación de inventarios en el almacén de mercancías de la Administración de Aduanas de Tacna, acceso adecuado a la información sobre las Unidades Orgánicas Operativas, así como información del Ministerio Público y el Poder Judicial, la asignación correcta de personal en apoyo de la gestión de almacenes y gestión eficiente del inventario de mercancías por parte de la administración actual. La materia prima, el suministro de información y la buena selección del personal de acuerdo al perfil que se necesita por área, son los medios que permiten agilizar la rotación del stock de mercancías, lo que también se relaciona con el presente trabajo de investigación ya que buscan menguar los riesgos, que afectan a la Superintendencia Nacional de Aduanas y Administración Tributaria. Churata R. (2014), concluye que los procedimientos técnico - administrativos que se aplicaron para la fiscalización de entrada y salida de mercancías no tuvieron efecto positivo en el control de transporte terrestre con la revisión de documentos e inspección física de vehículos y mercancías en el control aduanero. También el número de acciones operativas disminuyó en 3.5\% para el año 2010 y que de los bienes incautados en la Intendencia de Aduana de llo se utiliza ropa que tiene la mayor tasa de incautaciones en 2010 es el 56\% del total. En cuanto a las fallas técnico-administrativas que no permiten detectar el contrabando de 
mercancías en la Intendencia Aduanera - Ilo, considerando el Diagrama de Flujo, se ha detectado que en las acciones: origen de los vehículos, selección al azar, revisión de mercadería y confiscación de la mercadería de los vehículos por origen en el transporte de pasajeros y / o carga por la patrulla volante Montalvo, son los funcionarios de la Superintendencia Nacional de Aduanas y Administración Tributaria así como el personal de apoyo que está expuesto a contingencias en la pista (pisoteo, agresión, etc.) que pueden afectar su integridad física, así como la custodia de mercancías y bienes públicos ya que no tienen las condiciones físicas y de infraestructura. Churata R. menciona que los lineamientos propuestos generalmente son el resultado de la investigación y experiencia laboral en esta actividad aduanera y son: Reducir los márgenes de ganancia de los contrabandistas, reduciendo las tasas impositivas del comercio internacional a través de acuerdos comerciales y al mismo tiempo la Superintendencia Nacional de Aduanas e Impuestos. La administración y las fronteras nacionales deberían fortalecerse y modernizarse, supervisando y supervisando el transporte de mercancías mediante GPS y supervisando las paradas de vehículos. - Evaluaciones de las acciones realizadas o intervenciones para llevar a cabo una mejora continua. El nivel técnico (valoración de mercancías) de los oficiales debe ser alto para poder proceder de manera eficiente y rápida a las diferentes operaciones de despacho. Seguridad (personal) en el ejercicio de los controles. - La Comunicación, que incluye dos aspectos: comunicación interna y externa. Los procedimientos técnico - administrativos que se aplicaron para la fiscalización de entrada y salida de mercancías no tuvieron efecto positivo en el control de transporte terrestre, asimismo existe fallas técnicas que impiden detectar el contrabando, también propone que se reduzca las tasas tributarias por medio de tratados comerciales, modernizar la Superintendencia Nacional de Aduanas y de Administración Tributaria y que se emplee nuevas tecnologías en el supervisión de transporte de mercaderías, lo que también se relaciona con el presente trabajo de investigación porque en ambas investigaciones tienen como fin común la disminución de riesgos, que se cumpla con la normatividad vigente y no se perjudique a los intereses fiscales, económicos, la salud y la seguridad de los ciudadanos y que los usuarios no tengan inconveniente para trasladar y/o retirar sus mercancías de los recintos aduaneros. Pérez E y Arce J (2012), concluye que la teoría económica nos dice que existen dos razones por las que puede surgir el comercio entre países: la primera razón es que los países comercian porque son diferentes entre sí (en tecnología, dotaciones o preferencias) y pueden beneficiarse de ese comercio si cada uno produce y vende lo que sabe hacer relativamente mejor; la segunda razón se fundamenta en que existen fallas de mercado que hacen que existan Empresas Monopólicas y Oligopólicas que produzcan en economías de escala ,aprovechen economías externas, produzcan barreras tecnológicas, logrando mínimos costos de producción así como también existen organizaciones industriales que producen y comercializan a nivel interindustrial, cuyo comercio se da entre países desarrollados, logrando ganancias por este comercio y hacen que ante tales situaciones tengan que intervenir el estado con políticas comerciales ya sea en contra de empresas transnacionales o a favor de políticas estratégicas que promuevan un determinado sector. Según el Director General de la OMC, Pascal Lamí, la relación entre el comercio internacional y el valor del PIB mundial ha aumentado del 5,5\% en 1950 a más del 20\% en la actualidad, es así que El incremento del volumen, velocidad y complejidad del comercio internacional, estimulado por los avances tecnológicos que han revolucionado las prácticas globales del comercio, ha afectado significativamente la manera en que las autoridades aduaneras cumplen sus funciones, buscando lograr un balance apropiado entre facilitación y control regulatorio. La aplicación de los principios y procesos de gestión de riesgos proporciona los medios para lograr ese balance. Las Administraciones Aduaneras consideran que la inversión en el desarrollo y la modernización de las Aduanas ofrece ventajas considerables a los Gobiernos, ya que sienta las bases de una economía eficiente, de una protección adecuada de la sociedad y de unas infraestructuras sólidas. Pérez E y Arce J, indican que el desempeño de las Aduanas a escala nacional resulta fundamental para el cumplimiento de los objetivos del programa político de los Gobiernos. Una administración aduanera eficaz constituye un elemento crucial para la facilitación del comercio y, por consiguiente, para los objetivos y estrategias nacionales de desarrollo económico. Las Administraciones de Aduanas, sin embargo, están incorporando diversos mecanismos de control a fin de 
hacerlo más eficiente y efectivo, que no entrampe el normal flujo de comercio internacional. La aplicación de los principios y procesos de gestión de riesgos proporciona los medios para lograr un balance adecuado relacionado al control de riesgos.

\section{OBJETIVO}

Medir cómo la gestión de riesgos influye en la inspección de mercancías, inmovilización e incautación y control operativo en el Complejo Fronterizo "Santa Rosa", Centro de Control Aduanero "Tomasiri" y Puesto de Control Aduanero "Vila Vila" en la Intendencia de Aduana de Tacna, periodo 2016.

\section{METODOLOGÍA}

Investigación de diseño no experimental y descriptiva. La investigación se desarrollará en los 03 puestos de control más significativos (a nivel de afluencia de personas, equipajes y medios de transporte) de la Intendencia de Aduana de Tacna (Área Base: Complejo Fronterizo Santa Rosa, Centro de Control Aduanero Tomasiri, Puesto de Control Aduanero Vila Vila). La población estará conformada por los trabajadores (Especialistas) de los 03 puestos de control (36 trabajadores). Las encuestas se realizarán a los servidores quienes proporcionarán datos sobre la Gestión de Riesgos y las acciones de los puestos de Control. El instrumento es un cuestionario con 18 preguntas revisado por juicio de expertos.

\section{RESULTADOS}

En lo que se refiere a sí considera que cuenta con políticas y asignación de recursos adecuados, el 64\% indica que algunas veces. De lo que se entiende que no se cuenta con los recursos y políticas necesarios, debiendo efectuar los correctivos para su implementación. De acuerdo a la pregunta si considera que la administración tiene identificado los sectores de riesgo, el 55\% manifiesta que a veces, el $25 \%$ indica que casi nunca. De lo que se puede apreciar que falta mejorar la identificación de los sectores con mayores posibilidades de riesgo, debiendo mejorar e implementar los cambios necesarios en los procedimientos respectivos. Sobre la pregunta si se tienen identificados los fraudes para la toma de decisiones, el 36\% manifiesta que a veces, el 33\% indica que casi nunca y finalmente el 31\% revela que casi siempre. De lo que se observa que aún falta mejorar la identificación de posibilidad de fraude, debiendo implementar los correctivos necesarios. De acuerdo a la pregunta a si se cuenta con métodos para detectar engaños y ocultamientos, el $36 \%$ manifiesta que a veces, el $4 \%$ indica que casi siempre, el $22 \%$ revela que casi nunca y finalmente el $8 \%$ señala nunca. De lo que se entiende que falta mejorar los procedimientos para un mejor control y detección de bienes ocultos, así como realizar capacitaciones en estos temas. Sobre la pregunta si se considera que se vienen aplicando medidas correctivas de manera inmediata, el $42 \%$ revela que casi siempre, el 39\% manifiesta que a veces, el 19\% indica que casi nunca. De lo que se puede apreciar que los correctivos se vienen aplicando de manera parcial, debiendo efectuar los correctivos necesarios para su implementación. Sobre la pregunta relacionada a si los procedimientos con inteligencia experiencia y destreza son adecuados, el $42 \%$ indica que a veces. De lo que se puede apreciar que falta mejorar aun los procedimientos de experiencia y destreza para un control adecuado, debiendo efectuar los correctivos necesarios. En la pregunta a si considera que existe un nivel de control adecuado, el $47 \%$ indica que casi siempre. De lo que se puede apreciar que aún falta mejorar los procedimientos para contar con un nivel de control adecuado, debiendo establecer los correctivos necesarios. En lo relacionado a si se evalúan los riesgos de manera oportuna para la toma de decisiones, el $42 \%$ indica que casi nunca, el 33\% muestra que a veces. De lo que se puede apreciar que falta mejorar los procedimientos para una adecuada evaluación de riesgos, para la toma de decisiones, debiendo efectuar los correctivos necesarios. Con respecto a la pregunta si considera que se cuenta con el personal equipado eficazmente, el 53\% indica que a veces, el $28 \%$ muestra que casi nunca y finalmente el $19 \%$ revela que casi siempre. De lo que se puede entender que aún falta mejorar el equipamiento necesario para un mejor desempeño de funciones. De acuerdo a la pregunta a si se cuenta con los elementos logísticos necesarios para el control, 
el $47 \%$ indica que a veces, el $25 \%$ muestra que casi siempre, así como casi nunca y finalmente el 3\% revela que nunca.

En lo relacionado a si las programaciones de las inspecciones de mercancías son adecuadas, el 58\% indica que a veces. De lo que se puede entender que falta mejorar la programación en la inspección de mercancías, debiendo realizar las medidas correctivas necesarias. De lo que se puede apreciar que aún falta mejorar los mecanismos de control de los almacenes, debiendo establecer las acciones correctivas necesarios. En lo que se refiere a si las actas de inspección son entregadas de manera oportuna, el 3\% indica que casi nunca, el $3 \%$ indica que a veces, el $11 \%$ indica que casi siempre y finalmente del $83 \%$ muestra que siempre.

En lo que se refiere a si las actas de Inmovilización/Incautación son entregadas de manera oportuna, el 3\% indica que casi nunca, el $3 \%$ indica que a veces, el $11 \%$ indica que casi siempre y finalmente del $83 \%$ muestra que siempre. De lo que se puede apreciar que existe una correcta entrega de las actas de Inmovilización/Incautación. De acuerdo a la interrogante si se cumple con la normatividad relacionada a mercancías inmovilizadas o incautadas, el 3\% indica que casi nunca, el 3\% indica que a veces, el 11\% indica que casi siempre y finalmente del $83 \%$ muestra que siempre. Observándose que existe una correcta aplicación de la normatividad sobre la mercancía inmovilizada o incautada, pero que aún existen aspectos por mejorar. En lo que se refiere a si las medidas preventivas de inmovilización o incautación son adecuadas, $14 \%$ indica que casi siempre y finalmente el $80 \%$ muestra que siempre. De lo que se puede apreciar que, si bien es cierto, para los encuestados, la aplicación de medidas preventivas es muy aceptable, aún falta mejorar algunos aspectos. Sobre si se ejecutan acciones de control programadas de pasajeros, equipajes y vehículos (incluyendo Carga), 50\% indica que a veces, el $42 \%$ casi siempre y finalmente el $8 \%$ revela que casi nunca. De lo que se puede entender que falta mejorar la programación y ejecución de acciones de control programadas. En lo que se refiere a si se cuenta con medidas preventivas para la devolución de mercancías, 53\% indica que casi siempre, el 36\% revela que siempre y finalmente el $11 \%$ revela que a veces.

De lo que se puede apreciar que falta mejorar las herramientas necesarias para determinar el comiso o la devolución de mercancías, debiendo mejorar los procedimientos necesarios para su implementación. Sobre la pregunta si se previene delitos aplicando gestión de riesgo y correctivos de manera oportuna, $44 \%$ indica que a veces, el $42 \%$ revela que casi nunca, el 11\% indica que casi siempre y Finalmente el 3\% revela que nunca. Por lo que se puede deducir que falta implementar correctivos oportunos para mejorar el control para el traslado de las mercancías. (Tabla 01)

Tabla 1: Consolidado del instrumento aplicado

\begin{tabular}{|c|c|c|}
\hline \multicolumn{3}{|c|}{ Considera que cuenta con políticas y asignación de recursos adecuados } \\
\hline Casi nunca & 8 & $22.2 \%$ \\
\hline Algunas veces & 23 & $63.9 \%$ \\
\hline Casi siempre & 5 & $13.9 \%$ \\
\hline Total & 36 & $100.0 \%$ \\
\hline \multicolumn{3}{|c|}{ Considera que la administración tiene identificado los sectores de riesgo } \\
\hline Nunca & 1 & $2.8 \%$ \\
\hline Casi nunca & 9 & $25.0 \%$ \\
\hline A veces & 20 & $55.6 \%$ \\
\hline Casi siempre & 6 & $16.7 \%$ \\
\hline Total & 36 & $100.0 \%$ \\
\hline \multicolumn{3}{|c|}{ Se tiene identificado la posibilidad de fraudes para la toma de decisiones } \\
\hline Casi nunca & 12 & $33.3 \%$ \\
\hline A veces & 13 & $36.1 \%$ \\
\hline Casi siempre & 11 & $30.6 \%$ \\
\hline Total & 36 & $100.0 \%$ \\
\hline \multicolumn{3}{|c|}{ Se cuenta con métodos para detectar engaños y ocultamientos } \\
\hline Nunca & 3 & $8.3 \%$ \\
\hline Casi nunca & 8 & $22.2 \%$ \\
\hline A veces & 13 & $36.1 \%$ \\
\hline Casi siempre & 12 & $33.3 \%$ \\
\hline Total & 36 & $100.0 \%$ \\
\hline \multicolumn{3}{|c|}{ Considera que se vienen aplicando medidas correctivas de manera inmediata } \\
\hline Casi nunca & 7 & $19.4 \%$ \\
\hline A veces & 14 & $38.9 \%$ \\
\hline
\end{tabular}




\begin{tabular}{|c|c|c|}
\hline Casi siempre & 15 & $41.7 \%$ \\
\hline Total & 36 & $100.0 \%$ \\
\hline \multicolumn{3}{|c|}{ Procedimientos con inteligencia, experiencia y destreza son adecuados } \\
\hline Casi nunca & 11 & $30.6 \%$ \\
\hline A veces & 15 & $41.7 \%$ \\
\hline Casi siempre & 10 & $27.8 \%$ \\
\hline Total & 36 & $100.0 \%$ \\
\hline \multicolumn{3}{|c|}{ Considera que existe un nivel de control adecuado } \\
\hline Nunca & 1 & $2.8 \%$ \\
\hline Casi nunca & 5 & $13.9 \%$ \\
\hline A veces & 13 & $36.1 \%$ \\
\hline Casi siempre & 17 & $47.2 \%$ \\
\hline Total & 36 & $100.0 \%$ \\
\hline \multicolumn{3}{|c|}{ Se evalúan los criterios de riesgo oportunamente para la toma de decisiones } \\
\hline Casi nunca & 15 & $41.7 \%$ \\
\hline A veces & 12 & $33.3 \%$ \\
\hline Casi siempre & 9 & $25.0 \%$ \\
\hline Total & 36 & $100.0 \%$ \\
\hline \multicolumn{3}{|c|}{ Considera que se cuenta con el personal equipado eficazmente } \\
\hline Casi nunca & 10 & $27.8 \%$ \\
\hline A veces & 19 & $52.8 \%$ \\
\hline Casi siempre & 7 & $19.4 \%$ \\
\hline Total & 36 & $100.0 \%$ \\
\hline \multicolumn{3}{|c|}{ Elementos logísticos necesario para el control. } \\
\hline Nunca & 1 & $2.8 \%$ \\
\hline Casi nunca & 9 & $25.0 \%$ \\
\hline A veces & 17 & $47.2 \%$ \\
\hline Casi siempre & 9 & $25.0 \%$ \\
\hline \multicolumn{3}{|c|}{ La programación de las inspecciones de mercancías son adecuadas } \\
\hline Casi nunca & 9 & $25.0 \%$ \\
\hline A veces & 21 & $58.3 \%$ \\
\hline Casi siempre & 5 & $13.9 \%$ \\
\hline Siempre & 1 & $2.8 \%$ \\
\hline Total & 36 & $100.0 \%$ \\
\hline \multicolumn{3}{|c|}{ Las actas de inspección son entregadas de manera oportuna } \\
\hline Casi nunca & 1 & $2.78 \%$ \\
\hline A veces & 1 & $2.78 \%$ \\
\hline Casi siempre & 4 & $11.11 \%$ \\
\hline Siempre & 30 & $83.33 \%$ \\
\hline Total & 36 & $100.0 \%$ \\
\hline \multicolumn{3}{|c|}{ Las actas de inmovilización/incautación son informadas oportunamente } \\
\hline Casi nunca & 1 & $2.78 \%$ \\
\hline A veces & 1 & $2.78 \%$ \\
\hline Casi siempre & 4 & $11.11 \%$ \\
\hline Siempre & 30 & $83.33 \%$ \\
\hline Total & 36 & $100.0 \%$ \\
\hline \multicolumn{3}{|c|}{ Se cumple con la normatividad sobre mercancías } \\
\hline Casi nunca & 1 & $2.78 \%$ \\
\hline A veces & 1 & $2.78 \%$ \\
\hline Casi siempre & 4 & $11.11 \%$ \\
\hline Siempre & 30 & $83.33 \%$ \\
\hline Total & 36 & $100.0 \%$ \\
\hline \multicolumn{3}{|c|}{ Medidas preventivas de inmovilización o incautación adecuadas } \\
\hline Casi nunca & 1 & $2.8 \%$ \\
\hline A veces & 1 & $2.8 \%$ \\
\hline Casi siempre & 5 & $13.9 \%$ \\
\hline Siempre & 29 & $80.6 \%$ \\
\hline Total & 36 & $100.0 \%$ \\
\hline \multicolumn{3}{|c|}{ Se ejecutan acciones de control programadas de pasajeros, equipajes y vehículos } \\
\hline Casi nunca & 3 & $8.3 \%$ \\
\hline A veces & 18 & $50.0 \%$ \\
\hline Casi siempre & 15 & $41.7 \%$ \\
\hline Total & 36 & $100.0 \%$ \\
\hline & e me & \\
\hline Siempre & 13 & $36.1 \%$ \\
\hline Casi siempre & 19 & $52.8 \%$ \\
\hline A veces & 4 & $11.1 \%$ \\
\hline Total & 36 & $100.0 \%$ \\
\hline & nente & \\
\hline Nunca & 1 & $2.8 \%$ \\
\hline Casi nunca & 15 & $41.7 \%$ \\
\hline A veces & 16 & $44.4 \%$ \\
\hline Casi siempre & 4 & $11.1 \%$ \\
\hline Total & 36 & $100.0 \%$ \\
\hline
\end{tabular}




\section{DISCUSIÓN}

Del análisis realizado se puede observar que la gestión de riesgos puede influir significativamente en las acciones realizadas en los puestos de control de la Intendencia de Aduana de Tacna. Asimismo, el nivel estratégico, nivel táctico, nivel operativo en la gestión de riesgos podrían influir de manera significativa en las acciones de control en los puestos de la Intendencia. Los correctivos no se vienen aplicando oportunamente, debiendo efectuar los correctivos necesarios para su implementación. Chacón C. y Chávez M. (2010), manifiestan que la materia prima, el suministro de información y la buena selección del personal de acuerdo al perfil que se necesita por área, son los medios que permiten agilizar la rotación del stock de mercancías, lo que también se relaciona con el presente trabajo de investigación ya que buscan menguar los riesgos, que afectan a la Superintendencia Nacional de Aduanas y Administración Tributaria. También, Churata R. (2014), concluye que los procedimientos técnico administrativos que se aplicaron para la fiscalización de entrada y salida de mercancías no tuvieron efecto positivo en el control de transporte terrestre, asimismo existe fallas técnicas que impiden detectar el contrabando, también propone que se reduzca las tasas tributarias por medio de tratados comerciales, modernizar la Superintendencia Nacional de Aduanas y de Administración Tributaria y que se emplee nuevas tecnologías en el supervisión de transporte de mercaderías, lo que también se relaciona con el presente trabajo de investigación porque en ambas investigaciones tienen como fin común la disminución de riesgos, que se cumpla con la normatividad vigente y no se perjudique a los intereses fiscales, económicos, la salud y la seguridad de los ciudadanos y que los usuarios no tengan inconveniente para trasladar y/o retirar sus mercancías de los recintos aduaneros, lo que también se relaciona con el presente trabajo de investigación ya que buscan disminuir el delito aduanero a través de procedimientos. Pérez E y Arce J (2012), manifiestan que la aplicación de los principios y procesos de gestión de riesgos proporciona los medios para lograr un balance adecuado relacionado al control de riesgos, lo que también se relaciona con el presente trabajo de investigación.

En cada uno de los niveles estratégico, táctico y operativo, falta mejorar los procedimientos necesarios para controlar y fiscalizar el tráfico de mercancías; asimismo poder minimizar las posibles infracciones y delitos aduaneros. No se cuenta con las políticas y asignación de recursos adecuados, así mismo falta mejorar los mecanismos de control de los almacenes, la programación en la inspección de mercancías no es muy eficiente. Falta mejorar los procedimientos para contar con un nivel de control adecuado que permita mejorar el equipamiento necesario del personal para un mejor desempeño de sus funciones.

Se deberá mejorar, a través de un proceso de análisis y reestructuración, los procedimientos para controlar y fiscalizar el tráfico de mercancías; asimismo poder minimizar la posible comisión de infracciones y delitos aduaneros. Desde la administración se deberá mejorar la identificación de los sectores de riesgo, así como también mejorar procedimientos para un mejor control y detección de la comisión de infracciones y delitos aduaneros. Capacitar al personal sobre análisis de riesgo, manejo de información (inteligencia y contrainteligencia) y perfilamiento de pasajeros, equipajes y vehículos (incluyendo vehículos de carga).

\section{REFERENCIAS BIBLIOGRÁFICAS}

Agencia Española de Cooperación Internacional para el Desarrollo (2012) Implicaciones del Modelo OECD: Planificación Estratégica y su Articulación con la Gestión de Riesgo.

Chacón C y Chávez M (2010), Propuesta de mejora de la gestión de procesos del departamento de almacén de mercancías de la Intendencia de Aduana de Tacna - incremento de la rotación del stock Mercancías. [Tesis de maestría]. Universidad Peruana de Ciencias Aplicadas. 
Cedeño C, (2014) Beneficios de la gestión de riesgos en aduana [Trabajo monográfico] Universidad ECOTEC.

Churata R. (2014), Estrategias Técnico Administrativas para la disminución del delito aduanero: intendencia de aduana Ilo, periodo 2010. [Tesis de pregrado] Universidad Nacional del Altiplano. Puno, Perú

Castro P. \& Da Costa M, (2017) Guía para la gestión estratégica en aduanas. Recuperado de: http://www.captac-dr.org/c/document_library/-get_file?folderld=1944277\&name=DLFE-15164.

Control Aduanero (s/f) Convenio de Kyoto Directivas del anexo general. Cap. 6. Recuperado de: http:// www.wcoomd.org

Control-PG.01: Acciones de control aduanero. Publicada 23 de noviembre de 2016. Diario oficial "El Peruano" Lima. Perú.

Control-PE.00.01: Inmovilización-incautación y determinación legal de mercancías. Publicada el 30 de diciembre de 2016. Diario oficial "El Peruano" Lima. Perú.

Control-PE.01.03: Inspección de mercancías en zona primaria. Publicada 28 de agosto de 2013. Diario oficial "El Peruano" Lima. Perú.

Control-PG.01 (2016): Procedimiento general: acciones de control aduanero fiscalización y prevención del contrabando y control fronterizo Resolución: 0043-2016. Superintendencia Nacional de Aduanas y de Administración Tributaria. Diario oficial "El Peruano" Lima. Perú.

Decreto Legislativo № 1111. Ley Decreto Legislativo que modifica la Ley de los Delitos Aduaneros - Ley № 28008. Publicado el 29 de junio de 2012. Diario Oficial El Peruano. Lima - Perú.

Decreto Legislativo № 1053. Ley general de Aduanas. Publicado el 17 de junio de 2008. Diario Oficial El Peruano. Lima - Perú.

Decreto Supremo Nº10 - 2009 - EF.

Decreto Legislativo № 957. Código Procesal Penal, el numeral 7 de su Segunda Disposición Modificatoria y Derogatoria modifica el artículo 19‥ Publicado el 29 de julio de 2004. Diario Oficial El Peruano. Lima - Perú.

Decreto Legislativo № 1122. Decreto Legislativo que modifica la Ley General de Aduanas y la Ley de los Delitos Aduaneros. Publicado el 18 de julio de 2012. Diario Oficial El Peruano. Lima - Perú.

Directiva № 002-2014-SERVIR/GDSRH. Normas para la gestión del sistema administrativo de gestión de recursos humanos en las entidades públicas"

García A. (10 de febrero de 2017) Planeación Estratégica en Comercio Exterior. Recuperado de: http://www.estrategiaaduanera.mx/planeacion-estrategica-comercio-exterior/

Garret J. (2007) Control Aduanero. Recuperado de: http://www.comunidadandina.org/StaticFiles/201165195749libro_atrc_control.pdf

Hernández, S. (2010). Metodología de investigación científica. México: Mc Graw Hill/Interamericano.

Ley No28008. Ley de los delitos aduaneros. Publicado el 19 de junio de 2003. Diario Oficial El Peruano. Lima - Perú.

Ley Penal Tributaria Decreto Legislativo No. 813, Delitos Tributarios, Artículos 1 a 6. Defraudación Tributaria.

Ley № 28510. Ley que modifica el artículo 250 de la Ley № 28008, Ley de Delitos Aduaneros. Publicado el 17 de mayo de 2005. Diario Oficial El Peruano. Lima - Perú.

Ley № 29952. Ley de Equilibrio Financiero del Presupuesto del Sector Público para el año fiscal 2013. Publicado el 04 de diciembre de 2012. Diario Oficial El Peruano. Lima - Perú.

López, F. (2017) Acciones de control aduanero durante el despacho de exportación con acogimiento al Drawback en la IAAP, 2014 - 2015. Universidad Cesar Vallejo Recuperado de: http://repositorio.ucv.edu.pe/bitstream/handle/UCV/6516/L\%C3\%B3pez_SJF.pdf?sequence=1

Ministerio de Economía y Finanzas (10 de octubre de 2016) Definiciones. [Sitio Web]. Recuperado: https://www.mef.gob.pe/es/economia-internacional/politica-aduanera/definiciones 
Nueva estrategia (2017) Nueva estrategia de fiscalización aduanera. [Sitio Web] Recuperado de: http://www.aduanet.gob.pe/aduanas/informfis/Nueva_-Estrategia.htm

Pérez E y Arce J (2012), El control aduanero como sustento de la facilitación, mediante la gestión de riesgo en la oficina de oficiales de la aduana marítima del Callao. [Tesis de pregrado] Universidad Nacional de Ingeniería - UNI. Lima. Perú.

Resolución de Superintendencia Nacional Adjunta de Aduanas Nº0187-2013/SUNAT/300000INPCFAPG.01 (2013) Acciones de Control Aduanero. Diario Oficial El Peruano. Recuperado de: http://www.sunat.gob.pe/legislacion/proyectos-ta/2013/abril/050-413/INPCFA-PG-01.doc

República de Argentina (08 de Enero de 2018) Compendio de Gestión de Riesgos. Recuperado de: http://www.captac-dr.org/c/document_library/get_file?folderld=312918\&name=DLFE-825

Robbins, S. y Coulter, M. (2000), Administración. México. Prentice-Hall Hispanoamericana, S.A.

Superintendencia Nacional de Aduanas y de Administración Tributaria. (2016) Anexo-70-2016: Modificación del reglamento de organización y funciones de la superintendencia nacional de aduanas y de administración tributaria. [Sitio Web] Recuperado de: http://www.sunat.gob.pe/legislacion/superin/2016/anexo-070-2016.pdf

Superintendencia Nacional de Aduanas y de Administración Tributaria, Control-PE.01.03 - Inspección de mercancías en zona primaria, procedimiento específico: Resolución: 0208-2013-SUNAT-300000

Superintendencia Nacional de Aduanas y de Administración Tributaria, Control-PE.00.01: Inmovilizaciónincautación y determinación legal de mercancías, Resolución: 049-2016/SUNAT/5F0000.

Superintendencia Nacional de Aduanas y de Administración Tributaria, Control- PE.01.06: Control del valor declarado en importaciones de alto riesgo, Resolución: 00699-2010 